\title{
Exploring the provision of alcohol advice by clinicians to breast cancer patients
}

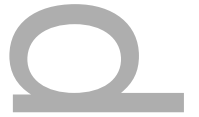

Phanthira Simapivapan ${ }^{\mathrm{a}}$, Allison Hodge ${ }^{\mathrm{a}, \mathrm{b}}$, Anna Boltong ${ }^{\mathrm{a}, \mathrm{b}}$

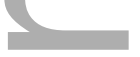

a The University of Melbourne; Parkville, Victoria 3010, Australia

${ }^{\mathrm{b}}$ Cancer Council Victoria; 615 St Kilda Road, Melbourne, Victoria 3004, Australia

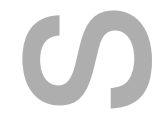

Corresponding author:

Dr Anna Boltong ${ }^{\mathrm{a}, \mathrm{b}}$

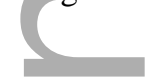

Head of Cancer Information and Support Services, Cancer Council Victoria; 615 St Kilda Road, Melbourne,

Victoria 3004, Australia

Tel: +61395146323

Email address: Anna.Boltong@ cancervic.org.au

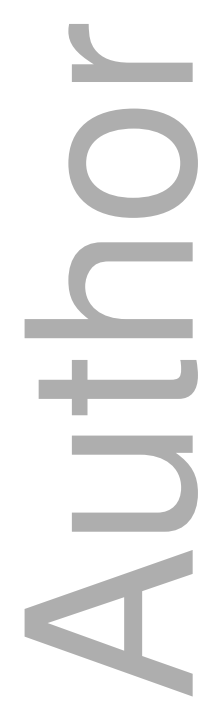

This is the author manuscript accepted for publication and has undergone full peer review but has not been through the copyediting, typesetting, pagination and proofreading process, which may lead to differences between this version and the Version of Record. Please cite this article as doi: $10.1111 /$ ecc. 12739

This article is protected by copyright. All rights reserved 
DR. PHANTHIRA SIMAPIVAPAN (Orcid ID : 0000-0002-3866-4705)

Article type : Original Article

$\underline{\text { Abstract }}$
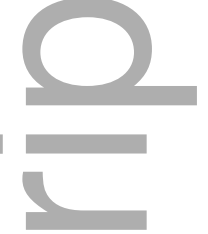

Interactions between clinicians and patients along the cancer trajectory provide an opportunity to deliver key messages regarding drinking behaviours and long term health. This study aimed to explore the extent and nature of clinician-patient discussions regarding alcohol intake and cancer outcomes in the clinical breast cancer setting, using a qualitative research design involving semi-structured interviews. Purposive sampling was used to recruit 27 breast cancer clinicians ( 8 dietitians, 9 breast care nurses, 10 oncologists) across Victoria, Australia. Interview data were analysed using descriptive statistics and a content analysis approach. Clinicians' knowledge of national alcohol recommendations was found to be inconsistent. Clinicians reported a lack of patient awareness of the link between alcohol and breast cancer. Current frameworks for assessing and advising on patient alcohol intake were felt to be impractical. The extent and nature of advice provided about alcohol was influenced by several patient and clinician factors. The provision of alcohol advice in the clinical breast cancer setting is not practiced systematically by any professional group. New approaches are needed to support patient education about alcohol intake and survivorship in the clinical oncology setting.

Keywords:

Alcohol

Breast cancer

Lifestyle advice

Health professionals

Survivorship

\section{Introduction}

It is estimated that one-third of all cancers in Australia and close to half of all new cancer diagnoses in the United States could be prevented through lifestyle modification (Colditz \& Sutcliffe, 2016; Whiteman et al., 2015). Alcohol consumption, in particular, has been associated with increased risk of a range of cancers, including oropharyngeal, oesophageal, colorectal and breast cancer (Bagnardi et al., 2015). For cancer prevention, the World Cancer Research Fund recommends healthy individuals who wish to consume alcohol abide by national alcohol guidelines, with similar recommendations for cancer survivors (Wiseman, 2008). In spite of increasing ease of access to health information largely via web-based technologies, the oncology 
healthcare team remain a key resource for people seeking post-diagnosis health advice (Finney Rutten et al., 2016). Research into the psychology of cancer survivors has shown that life threatening events, such as a diagnosis of breast cancer, can be a powerful motivator for patients to adopt a healthier lifestyle (Bluethmann et al., 2015). It is therefore within the remit of oncology health professionals to inform patients of actions they can take to maximise health outcomes following a cancer diagnosis.

A recent UK study examined the provision of clinician delivered lifestyle advice to oncology patients and found that awareness of lifestyle guidelines was low among cancer clinicians across multiple professional groups. Various barriers to advice provision were identified (Williams, Beeken, Fisher, \& Wardle, 2015). As yet, no equivalent study in Australia has explored the knowledge and behaviour of cancer clinicians regarding the provision of lifestyle advice to people with cancer. Alcohol intake is of particular relevance in the context of breast cancer, partly due to the strong link between alcohol and risk of primary breast cancer. Recent, though inconsistent, evidence also suggests amounts of alcohol intake below current public health recommendations are associated with increased risk of breast cancer recurrence (Kwan et al., 2013; Kwan et al., 2010; Simapivapan, Boltong, \& Hodge, 2016). This inconsistency in evidence may render specific advice provision on alcohol consumption challenging for clinicians, especially in the context of multiple clinical management and survivorship topics for discussion, including the benefits of alcohol consumption towards cardiovascular disease. It should be noted, however, that there is no consistent evidence supporting any benefit of postdiagnosis alcohol consumption in women previously diagnosed with breast cancer (Lowry, Kapphahn, Chlebowski, \& Li, 2016).

In order to investigate the extent and nature of clinician-patient discussions regarding alcohol intake and cancer outcomes in Australia, clinician interviews were undertaken to explore the following: 1) clinicians' understanding of public health recommendations regarding alcohol intake, 2) clinicians' attitudes and behaviours regarding alcohol advice for breast cancer patients, 3) patient and clinician factors that influence clinician-patient discussions about alcohol, and 4) barriers and opportunities for productive clinician-patient discussions about alcohol in the breast cancer context.

\section{Methods}

2.1. Study design and setting:

This study utilised a qualitative research design to explore clinician-patient discussions regarding alcohol in the clinical setting. Semi-structured interviews were used to gather data between February and March 2016. Purposive and snowball sampling was used to recruit oncology clinicians through a breast cancer speciality specific group within Cancer Council Victoria's clinical network and the relevant departments in which these clinicians worked.

2.2. Participants: 
Individuals were eligible to take part if they were: 1) a cancer specialist (medical oncologist, radiation oncologist, breast care nurse, dietitian) based in Victoria, Australia, and 2) currently in a position involving care of breast cancer patients. Individuals who met the inclusion criteria were sent an invitation email with study information, and then followed up by phone within the succeeding month. Individuals indicated if they were willing and available to participate in the study, or if they no longer met the inclusion criteria. Verbal consent was gained before each interview. Recruitment continued until data saturation was reached.

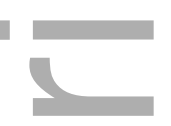

\subsection{Data collection:}

An interview guide (Table 1), including both open and closed questions, was developed specifically for this study, based on a UK questionnaire from a similar study exploring lifestyle advice provision by oncology clinicians (Williams et al., 2015). Important aspects of clinician-patient interactions addressed in the UK questionnaire were identified, and the relevant questions adapted to focus specifically on alcohol consumption. The interview guide was piloted with a breast care nurse, resulting in minor revisions to content ahead of implementation. Semi-structured interviews of approximately 30 minutes duration were conducted by one member of the research team, a trained medical student (PS) with no relationship to the participants, at the participants' workplace or by telephone. No audio or visual recording was used. Detailed notes were taken during the interview process, after which a copy was emailed to the participant for confirmation that views and interview responses were accurately captured.

\section{$<$ Insert Table $1>$}

\subsection{Data analysis:}

Interview transcripts were read and preliminary analysis conducted by one researcher (PS, medically trained), with subsequent analysis and refinement from all members of the research team $(\mathrm{AB}$, an experienced qualitative oncology researcher and dietitian; AH, a nutrition and cancer epidemiologist). Responses to closed, numerical questions were analysed using descriptive statistics and frequency counts. Responses to open ended questions were analysed using a content analysis approach as described by Gale et al (Gale, Heath, Cameron, Rashid, \& Redwood, 2013). Meaningful units of text under each interview question were identified, extracted and condensed. The resulting condensed meaning units were coded, and the coded data items collated and sorted into potential categories in tabular form. The appropriateness of categories and subcategories was discussed and refined until agreement was reached between all members of the research team. 
2.5. Ethical considerations:

Ethical approval for this study was obtained from Cancer Council Victoria's Institutional Research Review Committee (Project No: IER 1510).

\section{Results}

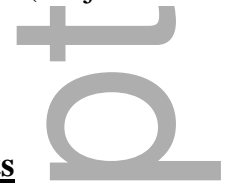

A total of 57 clinicians were approached to take part in this study. Of these clinicians, 27 (47\%) were interviewed. The remaining clinicians were either inaccessible by telephone on follow up (34\%), no longer met the inclusion criteria $(16 \%)$, were not interested or unavailable (3\%). Of the participants interviewed (Table 2), 8 were dietitians (30\%), 9 were breast care nurses (33\%) and 10 were oncologists $(37 \%$; 3 radiation oncologists, 7 medical oncologists).

$<$ Insert Table $2>$

\subsection{Knowledge of alcohol guidelines and frequency of clinician-patient discussions}

Responses to closed interview questions were quantified to assess clinicians' understanding of national alcohol recommendations (Interview question 1; Table 1) and frequency and patterns of clinician-patient discussions regarding alcohol (Interview questions 5, 6 and 7; Table 1).

\subsubsection{Clinicians' understanding of national alcohol recommendations}

Clinicians' knowledge of current national alcohol guidelines was inconsistent. Fourteen clinicians interviewed were able to recall recommendations on upper daily limits of alcohol intake consistent with Australia's National Health and Medical Research Council's (NHMRC) alcohol guidelines as being a maximum of two standard drinks per day (Australian Government National Health and Medical Research Council, 2015). Quantities of alcohol that constitute a 'standard drink' were not well known by clinicians of any professional group. One clinician was able to correctly recall standard drink volumes as $100 \mathrm{ml}$ of wine, $375 \mathrm{ml}$ of mid strength or $285 \mathrm{ml}$ of full strength beer and $30 \mathrm{ml}$ of spirits.

\subsubsection{Frequency and patterns of clinician-patient discussions regarding alcohol}

Clinicians reported that alcohol was not a common topic of discussion in consultations with breast cancer patients. Approximately one-third of the clinicians interviewed $(n=10)$ reported that they discussed alcohol with at least half the patients they saw. Around two-thirds of the clinicians interviewed $(n=18)$ reported that they were more likely than patients to initiate discussions about alcohol. Over half the oncologists interviewed $(n=6)$ explicitly stated that patients rarely or never initiated discussions about alcohol, while this was not reported by 
any breast care nurses or dietitians. Less than one-third of clinicians $(n=8)$ reported that breast cancer patients ever asked about alcohol in relation to cancer.

\subsection{Qualitative themes}

Key findings from clinicians' responses to open ended interview questions are described under three main qualitative themes with associated sub-themes (Table 3). Illustrative quotes provided are identified by the participant's profession (e.g. $\mathrm{D}=$ dietitian, $\mathrm{N}=$ breast care nurse, $\mathrm{O}=$ oncologist) and study number (e.g. $\mathrm{D} 1$, N3).

< Insert Table 3>

\subsubsection{Patient factors influencing clinician-patient discussions regarding alcohol}

\subsubsection{Lack of patient awareness of the link between alcohol and cancer:}

Clinicians reported a lack of awareness among cancer patients regarding the association between alcohol and cancer. Several oncologists commented on the surprised reactions of patients when informed of scientific evidence supporting alcohol as a risk factor for breast cancer, and when told explicitly that future excessive alcohol consumption may contribute to risk of a subsequent cancer. One oncologist (O1) stated that the most common perception patients had regarding alcohol and health risks was that alcohol caused accidents. This clinician also stated "the association between smoking and lung cancer was more widely understood" among patients. Another oncologist (O7) explained that most of their patients were from lower socioeconomic backgrounds and therefore often possessed limited knowledge of how lifestyle factors such as alcohol consumption are linked to cancer risk.

\subsubsection{Patient concerns regarding alcohol interactions with cancer treatment and cancer risk:}

Clinicians reported that, on the rare occasions when patients initiated discussions about alcohol, the most common concerns were the potential for adverse interactions with cancer treatment medications, followed by the association between alcohol consumption and the development of breast cancer. Clinicians explained that some patients, at the time of diagnosis, expressed concern about whether their alcohol intake may have contributed to the development of breast cancer. Patient concerns reportedly evolved during the treatment phase to include the potential dangers of consuming alcohol with different stages and types of cancer treatment. Common patient initiated questions reported by clinicians include: “Can I have alcohol?” (D7), “Can I still drink during chemotherapy?” (N2), and "Can I have alcohol and radiotherapy at the same time?” (O5). On completion of treatment, and in the transition to survivorship, some patients reportedly expressed concerns about alcohol and its effect on breast cancer recurrence. Common questions posed by patients to nurses and oncologists during this time include: "Does alcohol affect the likelihood of the cancer returning?" (N5) and, "Does drinking too much increase the risk of recurrence?" (O5). 


\subsubsection{Clinician factors influencing clinician-patient discussions regarding alcohol}

\subsubsection{The standard drink framework is unhelpful:}

Many clinicians across all three professional groups reported that standard drinks were not widely understood among patients, and clinicians who attempted to convey this concept in their discussions with patients often required practical and illustrative aids to support their explanations. One oncologist $(\mathrm{O} 4)$ stated that the concept of standard drinks is too specific, explaining that in consultations clinicians only required rough estimates of alcohol intake to gauge whether patients were consuming excessive amounts, and assessing alcohol consumption in terms of standard drinks is unnecessary and time consuming. Many oncologists expressed dissatisfaction with the concept of standard alcoholic drinks as an educational tool, stating that the concept is impractical and irrelevant to the consultation setting. Another oncologist (O2) reported purposely avoiding the model of standard drinks in discussions regarding alcohol because patients found the concept difficult to comprehend.

\subsubsection{Current alcohol guidelines are too generous:}

Many clinicians reported deliberately providing advice on alcohol consumption that was inconsistent with current public health guidelines, often recommending upper limits of alcohol intake that were lower than those stipulated by the NHMRC. In order to communicate recommendations on alcohol consumption to patients in an efficient and relatable manner, some clinicians reported adopting their own constructs for conveying appropriate levels of alcohol intake. One oncologist $(\mathrm{O} 2)$ stated that, although they were aware of existing alcohol guidelines and the concept of standard drinks, they instead chose to simplify their explanations regarding alcohol by advising cancer patients not to consume more than the equivalent of one bottle of wine per week. Other clinicians simply elected to apply lower limits regarding alcohol intake specifically for cancer patients. One breast care nurse (N3) reportedly recommends all breast cancer patients limit themselves to one glass of alcohol per day, followed by brief patient education on how research has shown alcohol is linked to the development of breast cancer. Another oncologist (O10) stated that current Australian alcohol guidelines "allow too much as far as cancer goes", and that recommendations on upper limits for people already diagnosed with breast cancer should be more consistent with the results of recent research studies which show alcohol intake as low as three to four standard drinks per week to be associated with increased risk of breast cancer recurrence.

3.2.2.3. Cancer prognosis and other patient characteristics:

When engaging in alcohol-related discussions, clinicians were often influenced by the personal and clinical characteristics of their patients. Four clinicians across the three professional groups (D5, N3, O1, O3) reported they were less likely to provide comprehensive alcohol advice with reference to specific upper limits to patients 
with poor cancer prognoses. One oncologist (O1) explained that this was because they preferred to refrain from pressuring palliative patients into "doing anything they didn't want to do with the time they had remaining". Two clinicians (D4, N3) reportedly emphasised strict upper limits for daily alcohol intake in discussions with postmenopausal or overweight women, as studies have shown these patient subgroups are at increased risk of cancer recurrence.

\subsubsection{Challenges to and opportunities for providing alcohol advice}

\subsubsection{Time and workload:}

Clinicians cited many barriers to the provision of alcohol advice. Many clinicians reported time constraints or an extensive list of higher priority issues that needed to be addressed during the allocated consultation time to be a major challenge in initiating clinician-patient discussions about alcohol. One oncologist (O7) stated that their main priority was to deal with cancer treatment and other medical issues, rarely allowing adequate time for discussions regarding lifestyle factors such as alcohol consumption. Several clinicians (D2, O7, O9) also expressed the opinion that advice provision was a lengthy and arduous process, adding to their reluctance to commence such discussions.

\subsubsection{Patient disinterest and resistance to advice:}

Clinicians across all professional groups reported the provision of alcohol advice to be challenging when interacting with patients who showed either disinterest in or aversion to the idea of discussing their alcohol consumption. Clinicians stated that this was often secondary to patients having a history of alcohol dependence. Clinicians were reportedly less likely to provide alcohol advice to such patients, either to avoid inadvertently impacting on clinician-patient rapport, or due to an expectation that the issue of alcohol misuse would be managed by another health professional. One dietitian (D6) reported being intimidated by the possibility of angering patients with a history of alcohol abuse, especially in situations such as home visits where safety might be a concern, and therefore chose not to discuss alcohol at all with these patients or used a very tentative approach.

\subsubsection{Everyone's business but no one's job:}

There was a wide difference of opinion across and within health professions regarding responsibility for informing patients of public health messages about alcohol. While several clinicians accepted responsibility for providing alcohol advice to patients, others actively or passively deferred this responsibility to another professional group or individual clinician. One oncologist (O5) stated they "did not mind" providing alcohol advice to patients, but also believed this task should ideally fall to dietitians. One dietitian (D7) expressed a belief that general practitioners should be responsible for routinely assessing patient alcohol intake and providing advice accordingly. A number of clinicians reported withholding alcohol advice with the expectation 
that the topic had been, or would be, discussed by other health professionals. One breast care nurse (N7) explicitly stated that they never initiated discussions about alcohol with patients, as this was 'medical advice' and considered the responsibility of oncologists or general practitioners.

\subsubsection{Changes to models of care:

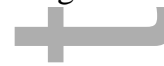

Some clinicians suggested that the routine integration of a designated care point within the cancer treatment trajectory where lifestyle factors and relevant modifications are discussed and planned for would be useful to ensure all oncology patients have the opportunity to discuss personal concerns and receive education regarding alcohol intake and cancer. One breast care nurse (N1) suggested that the establishment of a routine 'survivorship consultation', particularly in the late stages or after completion of cancer treatment, would provide a safe space where modifiable lifestyle factors such as alcohol consumption could be discussed with cancer patients in detail. One oncologist (O1) stated that such survivorship clinics should be run by health professionals whose sole responsibility would be to provide lifestyle advice and psychosocial assessments.

\section{Discussion}
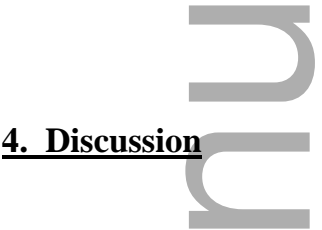

This study helped explore the fundamental interactions between clinicians and patients that make up the process of patient-centred care within the delicate scope of cancer survivorship, which is the premise on which the study was based. Interviews with breast cancer clinicians provided new information to assist understanding of both clinicians' attitudes and behaviours regarding alcohol advice provision, as well as patients' concerns about alcohol and cancer in the clinical breast cancer setting. Clinicians displayed inconsistent knowledge regarding current national alcohol intake recommendations, and expressed unfavourable views of existing frameworks used to assess, and advise on, alcohol intake. Patients were reported by clinicians to be generally naïve to the association between alcohol and cancer. The extent and nature of clinician-patient discussions regarding alcohol were inconsistent across and within different professional groups, and were influenced by many factors including patients' concerns and clinicians' motivations. Multiple challenges were reported to impede the advice provision process. The study findings reinforce that while reducing alcohol intake is believed to improve patient outcomes, the provision of directed advice to encourage this behavioural change in cancer patients is not yet established in routine healthcare.

Clinicians' inconsistent knowledge regarding alcohol guidelines, perceived patient disinterest and shortage of allotted time for consultations were common challenges to delivering lifestyle messages in line with current public health recommendations. This corroborates with findings from the UK study on which our study was based (Williams et al., 2015). Unlike the UK study, however, clinicians in the current study rarely cited patients being too frail or unwell as barriers to advice provision, possibly because of generally favourable survival rates among breast cancer patients, who formed the basis of this enquiry, compared to other heterogeneous patient samples (Kaplan, Malmgren, Atwood, \& Calip, 2015). The observation that the standard drinks construct is poorly understood by both clinicians and patients supports the results of two other studies which showed 
primary care patients and doctors of various specialities were generally unable to answer correctly when provided with questionnaires on standard drink volumes (Sprague \& Vinson, 2015; Welsh et al., 2014). A lack of knowledge or clarity surrounding public health guidelines may explain why some clinicians were reluctant to provide alcohol advice in their daily practice, or chose to avoid the concept of standard drinks in discussions about alcohol. Current national alcohol guidelines may be unclear or poorly publicised, and an alternative framework that conveys alcohol advice in a way that is easily comprehended by both patients and clinicians in the clinic setting is required.

Research into health education provides a robust framework from which the issues of patient education on the benefits of limiting alcohol consumption and subsequent behavioural modification can be further explored (Kok, van den Borne, \& Mullen, 1997). A recent online survey conducted in the UK to assess public knowledge of the link between alcohol and cancer found that only $18 \%$ of respondents were aware that alcohol is associated with increased risk of breast cancer (Buykx et al., 2015). This is consistent with comments made by clinicians in our study regarding the surprised reactions of patients when informed of this relationship. Research has shown that clinician-delivered lifestyle advice increases the likelihood of patients achieving subsequent sustained positive behavioural changes. A meta-analysis of independent studies exploring the effect of clinician delivered interventions to decrease alcohol intake concluded that brief interventions in the primary care setting consistently produced reductions in alcohol consumption in patients (Ballesteros, González-Pinto, Querejeta, \& Ariño, 2004). Multiple studies exploring the effect of health behaviour interventions in cancer patients have found both clinician-led face-to-face sessions and access to educational resources to result in, at the very least, short term improvement in certain modifiable lifestyle factors (Greenlee et al., 2016; Hebert et al., 2001). However, where the widespread application of personalised, face-to-face sessions are not considered feasible, other methods such as telephone counselling and the provision of quality and directed educational resources are viable alternatives that should continue to be explored (D. Anderson et al., 2017; Pierce et al., 2004).

In a population survey of cancer survivors and their social networks, over $80 \%$ of participants who considered lifestyle advice to be helpful also believed advice provision was a duty of doctors, although respondents were not specifically asked whether it was the doctors' role rather than that of other health professionals (Williams, Beeken, \& Wardle, 2013). However, oncologists we interviewed reported patients rarely or never initiated discussions on alcohol, while dietitians and breast care nurses made no such claims. This suggests that patients were more likely to initiate discussions about alcohol with dietitians and breast care nurses than oncologists, possibly due to a perception that consultations with these professions are more accommodating of lifestyle advice discussions (Stenner, Courtenay, \& Carey, 2011). Further complicating this matter is a lack of clarity among health professionals regarding whose role it is to provide alcohol advice. These issues have been found to apply more broadly with respect to other aspects of lifestyle modification, as well as among other cancer survivor populations, which were not explored in this study (A. S. Anderson, Caswell, Wells, \& Steele, 2013).

Current healthcare models do not support standardised provision of alcohol advice in the clinical setting, meaning patients are at risk of completing treatment and entering survivorship without having been actively advised on this point at any stage. Research has suggested that oncology patients are most receptive to lifestyle 
advice following cancer diagnosis, during treatment and immediately upon achieving remission, and so these time points in the cancer trajectory represent prime opportunities for such discussions to take place (DemarkWahnefried, Aziz, Rowland, \& Pinto, 2005; Karvinen, Bruner, \& Truant, 2015). Better delineation of professional roles and their designated responsibilities within the healthcare setting to assist the integration of alcohol advice into the cancer treatment trajectory is warranted.

\subsection{Strengths and limitations}

Although our-study included interviews with a range of health professionals across three key clinical groups involved in the care of breast cancer patients, there remain a number of limitations. First, the study sample did not include all professional groups, such as surgeons, and did not explore lifestyle factors beyond alcohol intake (e.g. smoking, physical activity, weight loss). Second, the study comprised a small sample size, of which opinions may not be generalisable to other health professionals. As a result, findings from this study are not considered to be an exhaustive representation of all clinicians' views, and instead represent a starting point for future, larger-scale studies exploring lifestyle advice provision to better inform supportive strategies that benefit both clinician and patient education.

\section{Conclusion \\ S. Conclusion}

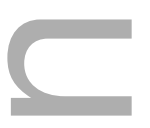

Provision of alcohol advice to breast cancer patients is not routine practice in the clinical oncology setting, and approaches vary within and between professional groups. Clinicians displayed inconsistent knowledge of national recommendations for alcohol intake, and expressed unfavourable views of current frameworks used to convey these recommendations. Despite this, clinician-patient discussions in the clinical setting are prime opportunities for patient education on alcohol intake and breast cancer, especially in light of observations by clinicians that the association between alcohol and cancer is poorly understood by patients. Findings from this study have helped to unravel some of the intricacies of clinician practices and patient behaviours surrounding discussions about alcohol in relation to breast cancer, and present a foundation on which future studies exploring the provision of lifestyle advice can build. This study will inform the design and testing of new educational tools regarding the contribution of alcohol to cancer outcomes for clinicians and patients alike. Future large-scale studies exploring clinician-delivered alcohol advice to breast cancer patients are warranted to determine whether findings from this study apply to the wider population, including professional groups not represented in the current sample.

\section{$\underline{\text { Acknowledgment }}$}

We would like to thank all the clinicians who generously donated their time to participate in this study. 


\section{$\underline{\text { Ethical approval }}$}

This study was approved by Cancer Council Victoria's Institutional Research Review Committee (project no: IER 1510) and has been performed in accordance with the ethical standards as laid down in the 1964 Declaration of Helsinki and its later amendments or comparable ethical standards.

I confirm all patient/personal identifiers have been removed or disguised so the patient/person(s) described are not identifiable and cannot be identified through the details of the story.

$\square$

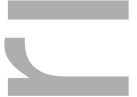

Conflicts of interest

None.

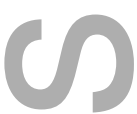

Funding

This research did not receive any specific grant from funding agencies in the public, commercial, or notfor-profit sectors.

\section{References}

Anderson, A. S., Caswell, S., Wells, M., \& Steele, R. J. C. (2013). Obesity and lifestyle advice in colorectal cancer survivors - how well are clinicians prepared? Colorectal Disease, 15, 949-957.

Anderson, D., Seib, C., Tjondronegoro, D., Turner, J., Monterosso, L., McGuire, A., . . McCarthy, A. L. (2017). The Women's wellness after cancer program: a multisite, single-blinded, randomised controlled trial protocol. BMC Cancer, 17, 98.

Australian Government National Health and Medical Research Council. (2015). Alcohol guidelines: reducing the health risks. Available from: https://www.nhmrc.gov.au/health-topics/alcohol-guidelines [last accessed 10 May 2016].

Bagnardi, V., Rota, M., Botteri, E., Tramacere, I., Islami, F., Fedirko, V., . . La Vecchia, C. (2015). Alcohol consumption and site-specific cancer risk: a comprehensive dose-response meta-analysis. British Journal of Cancer, 112, 580-593.

Ballesteros, J., González-Pinto, A., Querejeta, I., \& Ariño, J. (2004). Brief interventions for hazardous drinkers delivered in primary care are equally effective in men and women. Addiction, 99, 103-108.

Bluethmann, S. M., Basen-Engquist, K., Vernon, S. W., Cox, M., Gabriel, K. P., Stansberry, S. A., . . DemarkWahnefried, W. (2015). Grasping the 'teachable moment': time since diagnosis, symptom burden and health behaviors in breast, colorectal and prostate cancer survivors. Psycho-Oncology, 24, 1250-1257.

Buykx, P., Li, J., Gavens, L., Lovatt, M., Gomes de Matos, E., Holmes, J., . . Meier, P. (2015). An investigation of public knowledge of the link between alcohol and cancer. Available from: 
http://www.cancerresearchuk.org/sites/default/files/an investigation of public knowledge of the link between alcohol and cancer buykx et al.pdf [last accessed 22 May 2016].

Colditz, G. A., \& Sutcliffe, S. (2016). The Preventability of Cancer: Stacking the Deck. JAMA Oncology, 2, 1131-1133.

Demark-Wahnefried, W., Aziz, N. M., Rowland, J. H., \& Pinto, B. M. (2005). Riding the crest of the teachable moment: promoting long-term health after the diagnosis of cancer. Journal of Clinical Oncology, 23, 5814-5830.

Finney Rutten, L. J., Agunwamba, A. A., Wilson, P., Chawla, N., Vieux, S., Blanch-Hartigan, D., . . Hesse, B. W. (2016). Cancer-Related Information Seeking Among Cancer Survivors: Trends Over a Decade (2003-2013). Journal of Cancer Education, 31, 348-357.

Gale, N. K., Heath, G., Cameron, E., Rashid, S., \& Redwood, S. (2013). Using the framework method for the analysis of qualitative data in multi-disciplinary health research. BMC Medical Research Methodology, $13,1-8$.

Greenlee, H., Molmenti, C. L., Crew, K. D., Awad, D., Kalinsky, K., Brafman, L., . . Hershman, D. L. (2016). Survivorship care plans and adherence to lifestyle recommendations among breast cancer survivors. Journal of Cancer Survivorship, 10, 956-963.

Hebert, J. R., Ebbeling, C. B., Olendzki, B. C., Hurley, T. G., Ma, Y., Saal, N., . . Clemow, L. (2001). Change in Women's Diet and Body Mass Following Intensive Intervention for Early-stage Breast Cancer. Journal of the American Dietetic Association, 101, 421-431.

Kaplan, H. G., Malmgren, J. A., Atwood, M. K., \& Calip, G. S. (2015). Effect of treatment and mammography detection on breast cancer survival over time: 1990-2007. Cancer, 121, 2553-2561.

Karvinen, K., Bruner, B., \& Truant, T. (2015). The Teachable Moment After Cancer Diagnosis: Perceptions From Oncology Nurses. Oncology Nursing Forum, 42, 602-609.

Kok, G., van den Borne, B., \& Mullen, P. D. (1997). Effectiveness of health education and health promotion: meta-analyses of effect studies and determinants of effectiveness. Patient Education and Counseling, $30,19-27$.

Kwan, M. L., Chen, W. Y., Flatt, S. W., Weltzien, E. K., Nechuta, S. J., Poole, E. M., . . Caan, B. J. (2013). Postdiagnosis alcohol consumption and breast cancer prognosis in the after breast cancer pooling project. Cancer Epidemiology, Biomarkers \& Prevention, 22, 32-41.

Kwan, M. L., Kushi, L. H., Weltzien, E., Tam, E. K., Castillo, A., Sweeney, C., \& Caan, B. J. (2010). Alcohol consumption and breast cancer recurrence and survival among women with early-stage breast cancer: The life after cancer epidemiology study. Journal of Clinical Oncology, 28, 4410-4416.

Lowry, S. J., Kapphahn, K., Chlebowski, R., \& Li, C. I. (2016). Alcohol Use and Breast Cancer Survival among Participants in the Women's Health Initiative. Cancer Epidemiology, Biomarkers \& Prevention, 25, 1268-1273.

Pierce, J. P., Newman, V. A., Flatt, S. W., Faerber, S., Rock, C. L., Natarajan, L., . . Group, L. S. (2004). Telephone Counseling Intervention Increases Intakes of Micronutrient- and Phytochemical-Rich Vegetables, Fruit and Fiber in Breast Cancer Survivors. The Journal of Nutrition, 134, 452-458. 
Simapivapan, P., Boltong, A., \& Hodge, A. (2016). To what extent is alcohol consumption associated with breast cancer recurrence and second primary breast cancer?: A systematic review. Cancer Treatment Reviews, 50, 155-167.

Sprague, D. J., \& Vinson, D. C. (2015). Patient perceptions of risky drinking: knowledge of daily and weekly low-risk guidelines and standard drink sizes. Substance Abuse.

Stenner, K. L., Courtenay, M., \& Carey, N. (2011). Consultations between nurse prescribers and patients with diabetes in primary care: A qualitative study of patient views. International Journal of Nursing Studies, $48,37-46$.

Welsh, C., Earley, K., Delahanty, J., Wright, K. S., Berens, T., Williams, A. A., . . DiClemente, C. C. (2014). Residents' knowledge of standard drink equivalents: implications for screening and brief intervention for at-risk alcohol use. The American Journal on Addictions, 23, 194-196.

Whiteman, D. C., Webb, P. M., Green, A. C., Neale, R. E., Fritschi, L., Bain, C. J., . . Carey, R. N. (2015). Cancers in Australia in 2010 attributable to modifiable factors: summary and conclusions. Australian and New Zealand journal of public health, 39, 477-484.

Williams, K., Beeken, R. J., Fisher, A., \& Wardle, J. (2015). Health professionals' provision of lifestyle advice in the oncology context in the United Kingdom. European Journal of Cancer Care, 24, 522-530.

Williams, K., Beeken, R. J., \& Wardle, J. (2013). Health behaviour advice to cancer patients: the perspective of social network members. British Journal of Cancer, 108, 831-835.

Wiseman, M. (2008). The Second World Cancer Research Fund/American Institute for Cancer Research Expert Report. Food, Nutrition, Physical Activity, and the Prevention of Cancer: A Global Perspective. Proceedings of the Nutrition Society, 67, 253-256.

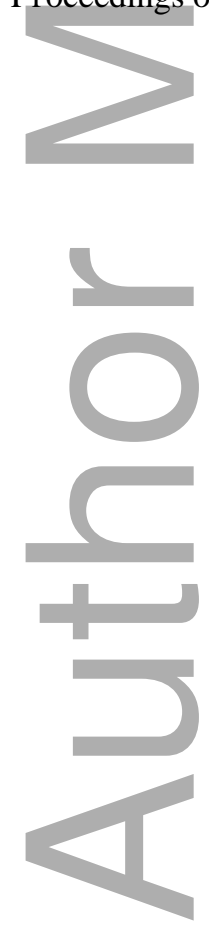


Table 1: Interview guide

1. Are you familiar with any guidelines or recommendations regarding alcohol intake for the general public (yes/no)? Can you state the contents of those guidelines?

2. Do you refer to these guidelines/recommendations in your interactions with patients, and does your advice on alcohol intake differ for a person diagnosed with breast cancer as compared to other cancer types? Why/Why not?

3. Do you consider risk of breast cancer recurrence in your discussions?

4. How would you describe what a standard alcoholic drink is to a patient?

5. In general, what proportion of your patients do you find involve discussion regarding alcohol intake (answer in percentage)? What usually precipitates these discussions?

6. Of these discussions on alcohol, what proportion are patients asking you for information (answer in percentage)? What are usually their main concerns? What specific questions do they ask you?

7. What proportion of these discussions are you the one to initiate discussion on alcohol (answer in percentage)? What are your main concerns when you approach these patients?

8. What circumstances make it challenging for you to give advice on alcohol intake? How often do these circumstances occur?

9. When you provide patients with information regarding alcohol intake, does your recommendation or approach vary according to their risk factors? If so, then in what way?

10. What methods do you use to supply your patients with information regarding alcohol intake? What is your preferred method?

11. What resource do you as a professional primarily refer to when searching for information on modifiable lifestyle factors in cancer?

12. If there was one thing you could have to make it easier for you to discuss modifiable lifestyle factors to your cancer patients, what would it be?

13. What about specifically in relation to recommendations on alcohol intake? What would be most helpful?

14. Whose role do you believe the provision of alcohol and general lifestyle advice falls under?

Table 2: Participant characteristics $(\mathrm{n}=27)$

\begin{tabular}{lllll}
\hline Variable & Dietitians & Breast care nurses & Oncologists & Overall \\
& & & & N (\%) \\
\hline Gender & 1 & 0 & 5 & $6(22)$ \\
Male & 7 & 9 & 5 & $21(78)$ \\
Female & & & & \\
Age range (years) & & & & \\
\hline
\end{tabular}




\begin{tabular}{|c|c|c|c|c|}
\hline $20-29$ & 3 & 0 & 0 & $3(11)$ \\
\hline 30-39 & 3 & 0 & 1 & $4(15)$ \\
\hline $40-49$ & 0 & 5 & 3 & $8(30)$ \\
\hline 50-59 & 2 & 4 & 4 & $10(37)$ \\
\hline 60-69 & 0 & 0 & 2 & $2(7)$ \\
\hline \multicolumn{5}{|c|}{ Professional experience in oncology (years) } \\
\hline Mean \pm SD & $5.8 \pm 4.9$ & $19.9 \pm 8.4$ & $22.9 \pm 9.0$ & $16.8 \pm 10.5$ \\
\hline \multicolumn{5}{|c|}{ Frequency of dealing with breast cancer patients } \\
\hline Frequently ( $\geq 1$ patient per week) & 3 & 9 & 10 & $22(81)$ \\
\hline Sometimes ( $\geq 1$ patient per month) & 2 & 0 & 0 & $2(7)$ \\
\hline Rarely ( $\geq 1$ patient per 3 months) & 3 & 0 & 0 & $3(11)$ \\
\hline
\end{tabular}

Table 3: Themes and sub-themes arising from analysis of clinician interviews

1. Patient factors influencing clinician-patient discussions regarding alcohol

- Lack of patient awareness of the link between alcohol and cancer

- Patient concerns regarding alcohol interactions with cancer treatment and cancer risk

2. Clinician factors influencing clinician-patient discussions regarding alcohol

- $\quad$ The standard drink framework is unhelpful

- Current alcohol guidelines are too generous

- Cancer prognosis and other patient characteristics

3. Challenges to and opportunities for providing alcohol advice

- $\quad$ Time and workload

- Patient disinterest and resistance to advice

- Everyone's business but no one's job

- Changes to models of care 


\section{University Library}

\section{- M M N E R VA A gateway to Melbourne's research publications}

Minerva Access is the Institutional Repository of The University of Melbourne

Author/s:

Simapivapan, P;Hodge, A;Boltong, A

Title:

Exploring the provision of alcohol advice by clinicians to breast cancer patients

Date:

2018-01-01

Citation:

Simapivapan, P., Hodge, A. \& Boltong, A. (2018). Exploring the provision of alcohol advice by clinicians to breast cancer patients. EUROPEAN JOURNAL OF CANCER CARE, 27 (1), https://doi.org/10.1111/ecc.12739.

Persistent Link:

http://hdl.handle.net/11343/293220 